\section{Effects of Prohexadione-Calcium on Fruit Size and Return Bloom in Pear}

\author{
David Sugar \\ Oregon State University, Southern Oregon Research and Extension Center, \\ 569 Hanley Road, Medford, OR 97502
}

\author{
Don C. Elfving \\ Washington State University, Tree Fruit Research and Extension Center, 1100 \\ North Western Avenue, Wenatchee, WA 98801
}

\author{
Eugene A. Mielke \\ Oregon State University, Mid-Columbia Agricultural Research and Extension \\ Center, 3005 Experiment Station Drive, Hood River, OR 97031
}

Additional index words. Pyrus communis, Apogee, plant growth regulators, gibberellin inhibitors

\begin{abstract}
The effects of prohexadione-calcium (P-Ca) on fruit size and return bloom in three pear cultivars were evaluated in Medford and Hood River, Ore., and in Cashmere, Wash. A variety of treatment dosages and timings was applied to 4- and 5-year-old trees in 2 years of study. Fruit weight of 'Bosc' and 'Red Anjou' pears was not affected by P-Ca treatments at any location in either year. However, decreased weight of 'Bartlett' pear fruit was associated with all P-Ca treatments in 1999 in Medford except for $83 \mathrm{ppm}$ applied at 2.5 to $6.0 \mathrm{~cm}$ shoot growth (first treatment) plus 2, 4, 6, and 8 weeks after first treatment (WAFT) and $125 \mathrm{ppm}$ applied at 2.5 to $6.0 \mathrm{~cm}$ growth plus 4 WAFT. 'Bartlett' fruit weight was reduced in Medford in 2000 by all treatments except $125 \mathrm{ppm}$ applied at 2.5 to $6.0 \mathrm{~cm}$ growth plus 4, 8, and 12 WAFT. In Cashmere in 2000, mean weight of 'Bartlett' and 'd'Anjou' fruit was reduced by treatments with 83 or $125 \mathrm{ppm}$ applied at 2.5 to $6.0 \mathrm{~cm}$ growth plus 2,4 , and 6 WAFT and of 'Bosc' pear by all treatments that included more than a single application of P-Ca. Crop load was not significantly different among treatments at any location. Return bloom in the year following P-Ca treatment was reduced in 'Bosc' pears by some to most treatments at all locations in both years. In contrast, return bloom was reduced in 'Bartlett' and 'Anjou' pears only in Hood River in 1999.
\end{abstract}

Prohexadione-calcium (P-Ca) received federal registration in the United States in 2000 for vegetative growth reduction and management of fire blight in apple shoots under the trade name Apogee (BASF Corp., 2003). $\mathrm{P}-\mathrm{Ca}$ inhibits shoot elongation by inhibiting the biosynthesis of growth-active gibberellins (Evans et al., 1999). Inhibition of shoot elongation has been demonstrated in apple (Greene, 1999; Unrath, 1999) and sweet cherry (Elfving et al., 2003) trees, but shoot growth was not inhibited in peach at the concentrations and timing tested (Byers and Yoder, 1999). In pear trees, $\mathrm{P}-\mathrm{Ca}$ solutions can inhibit shoot elongation when applied during early shoot growth (Basak and Rademacher, 2000; Costa et al., 2000; Elfving et al., 2002, 2003b).

In apple, $\mathrm{P}-\mathrm{Ca}$ applications increased fruit set and correspondingly reduced average individual fruit weight. Return bloom in the year following treatment declined with increasing $\mathrm{P}-\mathrm{Ca}$ dosage, and appeared to be inversely related to fruit set in the year of treatment (Greene, 1999). In contrast, Costa et al. (2000) reported a tendency for both increased fruit size and enhanced return bloom in apple. P-Ca applications alone did not affect return bloom in sweet cherry, although return bloom increased when ethephon was combined with $\mathrm{P}-\mathrm{Ca}$ in

Received for publication 7 July 2003. Accepted for publication 14 Jan. 2004. the earliest treatment (Elfving et al., 2003a). No effect on fruit size was reported following P-Ca applications to 'Rosemarie' pear in South Africa (Theron et al., 2002).

The objective of the present study was to evaluate the effects of P-Ca on fruit size and return bloom in three pear cultivars in each of three pear-growing regions of the Pacific Northwest.

\section{Materials and Methods}

Research plots were established at the Southern Oregon Research and Extension Center, Medford, the Mid-Columbia Agricultural Research and Extension Center, Hood River, Ore., and in commercial pear orchards in Cashmere, Wash., near the Washington Tree Fruit Research and Extension Center, Wenatchee. 'Bartlett' and 'Bosc' pear cultivars were included at all sites; 'Anjou' was included at Hood River and Cashmere while 'Red Anjou' (Gebhard strain) was used at Medford. In Medford and Hood River, all trees were on $\mathrm{OHxF} 97$ rootstock and were four years old when the study began in 1999 . In the 1999 trials in Cashmere, 'Bosc'/OHxF97, 'Anjou'/OHxF87 and 'Bartlett'/OHxF97 trees were in their fourth leaf. In the 2000 trial in Cashmere, 'd'Anjou trees were on $\mathrm{OHxF} 69$, 87 and 97, 'Bartlett' trees were on $\mathrm{OHxF} 40$, 69, 87 and 97, and 'Bosc' trees were on $\mathrm{OHxF}$
40, 87, and 97. All trees were in their fourth leaf at the start of the trial.

A variety of different P-Ca dosages and timings was applied in 1999, with dosages ranging from 83 to $600 \mathrm{ppm}$ (Tables 1, 3, and $5)$. The earliest application timing occurred at 2.5 to $6.0 \mathrm{~cm}$ of new shoot growth (= first treatment), and timings ranged up to 12 weeks after first treatment (WAFT). In 2000, treatment dosages and timings were standardized among study sites, with some variation. Treatments were applied to single-tree replications in a completely randomized design with six replications per treatment (Medford), a randomized complete-block design with four replications per treatment (Hood River), and a split-plot treatment arrangement in randomized complete-block design with nine to twelve replications per treatment (Cashmere). In the latter trial, rootstock was the main plot and $\mathrm{P}-\mathrm{Ca}$ treatments constituted the split plot.

At harvest in each year, the fruit on each tree were counted and weighed. In the spring following each year of treatment, the number of flower clusters (return bloom) per tree was counted. In Cashmere, return bloom was determined on whole trees for 'Bosc' in 1999, but for the other cultivars in 1999 and for all cultivars in 2000, return bloom was counted on one scaffold limb system per tree (Forshey and Elfving, 1979). Trees of 'Anjou' in Hood River and 'Bosc' in Cashmere were nonbearing in 1999 when the study began.

Mean values in data from Medford and Hood River were separated by Fisher's LSD where significance was identified by ANOVA $(P<0.05)$ using Minitab software (Minitab Inc., State College, Pa.). An exception was return bloom data from Hood River, which was similarly analyzed using SAS (SAS Inst., Cary, N.C.). Mean values in data from Cashmere were separated using the Waller/Duncan Bayesian $\mathrm{k}$ ratio test where significance was identified by ANOVA $(P<0.05)$ using SAS.

\section{Results}

Decreased weight of 'Bartlett' pear fruit was associated with all P-Ca treatments in 1999 in Medford except for $83 \mathrm{ppm}$ applied at 2.5 to $6.0 \mathrm{~cm}$ growth plus $2,4,6$, and 8 WAFT and $125 \mathrm{ppm}$ applied at 2.5 to $6.0 \mathrm{~cm}$ growth plus 4 WAFT (Table 1). 'Bartlett' fruit weight was reduced in Medford in 2000 by all treatments except $125 \mathrm{ppm}$ applied at $2.5-6.0 \mathrm{~cm}$ growth plus 4, 8, and 12 WAFT (Table 2). 'Bartlett' fruit weight was also reduced by treatments with 83 or $125 \mathrm{ppm}$ applied at 2.5 to $6.0 \mathrm{~cm}$ growth plus 2, 4, and 6 WAFT in Cashmere in 2000 (Table 6). 'Bartlett' fruit weight was not affected by P-Ca treatments in Cashmere in 1999 (Table 5) or in either year in Hood River (Tables 3 and 4). Fruit weight of 'Bosc', 'Anjou', and 'Red Anjou' pears was not reduced by P-Ca treatments at the Oregon sites in either year but was reduced in all cultivars by P-Ca treatments in Cashmere in 2000 (Tables 1-6). In all varieties, locations, and years, the crop load (number of fruit per tree) did not differ significantly among treatments (Tables 1-6). 
Table 1. Effect of various treatment dosages and timings of prohexadione-calcium applications on fruit weight, crop load, and return bloom in three pear cultivars. Medford, Oregon, 1999.

\begin{tabular}{|c|c|c|c|c|c|c|c|c|c|}
\hline \multirow[b]{2}{*}{ Treatment $^{\mathrm{z}}$} & \multicolumn{3}{|c|}{ Bartlett } & \multicolumn{3}{|c|}{ Bosc } & \multicolumn{3}{|c|}{ Red Anjou } \\
\hline & $\begin{array}{c}\text { Fruit wt } \\
(\mathrm{g})^{\mathrm{y}}\end{array}$ & $\begin{array}{l}\text { Fruit/ } \\
\text { tree }\end{array}$ & $\begin{array}{l}\text { Return } \\
\text { bloom }^{\mathrm{x}}\end{array}$ & $\begin{array}{l}\text { Fruit wt } \\
\text { (g) }\end{array}$ & $\begin{array}{l}\text { Fruit/ } \\
\text { tree }\end{array}$ & $\begin{array}{l}\text { Return } \\
\text { bloom }\end{array}$ & $\begin{array}{l}\text { Fruit wt } \\
\text { (g) }\end{array}$ & $\begin{array}{c}\text { Fruit/ } \\
\text { tree }\end{array}$ & $\begin{array}{l}\text { Return } \\
\text { bloom }\end{array}$ \\
\hline Untreated & $204.7 \mathrm{a}$ & $102.0 \mathrm{a}$ & $348 \mathrm{a}$ & $240.3 \mathrm{a}$ & $52.8 \mathrm{a}$ & $128 \mathrm{a}$ & $204.8 \mathrm{a}$ & $31.8 \mathrm{a}$ & $238 \mathrm{a}$ \\
\hline $250 \mathrm{ppm} 2.5-6.0 \mathrm{~cm}$ & $176.7 \mathrm{bc}$ & $117.8 \mathrm{a}$ & $281 \mathrm{a}$ & 238.9 a & $61.5 \mathrm{a}$ & $33 \mathrm{bc}$ & $237.8 \mathrm{a}$ & $27.0 \mathrm{a}$ & $139 \mathrm{a}$ \\
\hline 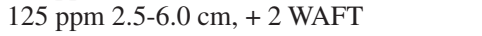 & $170.5 b c$ & $136.3 \mathrm{a}$ & $285 \mathrm{a}$ & $248.0 \mathrm{a}$ & $53.8 \mathrm{a}$ & $34 \mathrm{bc}$ & $218.2 \mathrm{a}$ & $44.5 \mathrm{a}$ & $136 \mathrm{a}$ \\
\hline $250 \mathrm{ppm} 2.5-6.0 \mathrm{~cm},+2 \mathrm{WAFT}$ & $171.7 \mathrm{bc}$ & $140.8 \mathrm{a}$ & $338 \mathrm{a}$ & $240.1 \mathrm{a}$ & $49.5 \mathrm{a}$ & $33 \mathrm{bc}$ & $207.2 \mathrm{a}$ & $43.3 \mathrm{a}$ & $90 \mathrm{a}$ \\
\hline $125 \mathrm{ppm} 2.5-6.0 \mathrm{~cm},+2,4,6$ WAFT & $158.3 \mathrm{c}$ & $135.0 \mathrm{a}$ & $275 \mathrm{a}$ & $244.8 \mathrm{a}$ & $41.8 \mathrm{a}$ & $17 \mathrm{c}$ & $202.6 \mathrm{a}$ & $34.8 \mathrm{a}$ & $106 \mathrm{a}$ \\
\hline $125 \mathrm{ppm} 2.5-6.0 \mathrm{~cm},+4$ WAFT & $187.3 \mathrm{ab}$ & $119.0 \mathrm{a}$ & $276 \mathrm{a}$ & $251.5 \mathrm{a}$ & $44.8 \mathrm{a}$ & $75 \mathrm{~b}$ & $208.8 \mathrm{a}$ & $36.3 \mathrm{a}$ & $104 \mathrm{a}$ \\
\hline 125 ppm $2.5-6.0 \mathrm{~cm},+250$ ppm 4 WAFT & $169.3 \mathrm{bc}$ & $121.0 \mathrm{a}$ & $361 \mathrm{a}$ & $261.3 \mathrm{a}$ & $48.3 \mathrm{a}$ & $48 \mathrm{bc}$ & $229.4 \mathrm{a}$ & $13.5 \mathrm{a}$ & $115 \mathrm{a}$ \\
\hline $83 \mathrm{ppm} 2.5-6.0 \mathrm{~cm},+2,4,6,8 \mathrm{WAFT}$ & $205.0 \mathrm{a}$ & $121.0 \mathrm{a}$ & $293 \mathrm{a}$ & $254.0 \mathrm{a}$ & $36.3 \mathrm{a}$ & $42 \mathrm{bc}$ & $243.8 \mathrm{a}$ & $6.3 \mathrm{a}$ & $125 \mathrm{a}$ \\
\hline
\end{tabular}

${ }^{2}$ First treatment was applied at $2.5-6.0 \mathrm{~cm}$ of new shoot growth. Subsequent applications were made at the same or different dosage at the indicated number of weeks after first treatment (WAFT).

${ }^{\mathrm{y}}$ Values followed by the same letter are not significantly different $(P>0.05)$ according to the Waller-Duncan Bayesian k ratio test $(P>0.05)$

${ }^{x}$ Average number of flower clusters per tree in 2000 following treatment in 1999.

Table 2. Effect of various treatment dosages and timings of prohexadione-calcium applications on fruit weight, crop load, and return bloom in three pear cultivars, Medford, Ore., 2000.

\begin{tabular}{|c|c|c|c|c|c|c|c|c|c|}
\hline \multirow[b]{2}{*}{ Treatment $^{2}$} & \multicolumn{3}{|c|}{ Bartlett } & \multicolumn{3}{|c|}{ Bosc } & \multicolumn{3}{|c|}{ RedAnjou } \\
\hline & $\begin{array}{l}\text { Fruit wt } \\
(\mathrm{g})^{\mathrm{y}}\end{array}$ & $\begin{array}{c}\text { Fruit/ } \\
\text { tree }\end{array}$ & $\begin{array}{l}\text { Return } \\
\text { bloom }^{\mathrm{x}}\end{array}$ & $\begin{array}{c}\text { Fruit wt } \\
(\mathrm{g})\end{array}$ & $\begin{array}{c}\text { Fruit/ } \\
\text { tree }\end{array}$ & $\begin{array}{l}\text { Return } \\
\text { bloom }\end{array}$ & $\begin{array}{l}\text { Fruit wt } \\
(\mathrm{g})\end{array}$ & $\begin{array}{c}\text { Fruit/ } \\
\text { tree }\end{array}$ & $\begin{array}{l}\text { Return } \\
\text { bloom }\end{array}$ \\
\hline Untreated & $200.1 \mathrm{a}$ & $119 \mathrm{a}$ & $86.8 \mathrm{a}$ & $292.1 \mathrm{a}$ & $58.0 \mathrm{a}$ & $107.5 \mathrm{a}$ & $173.0 \mathrm{a}$ & $51.3 \mathrm{a}$ & $222.7 \mathrm{a}$ \\
\hline $83 \mathrm{ppm} 2.5-6.0 \mathrm{~cm},+2,4,6,8 \mathrm{WAFT}$ & $166.2 \mathrm{bc}$ & $155 \mathrm{a}$ & $115.3 \mathrm{a}$ & $283.4 \mathrm{a}$ & $79.5 \mathrm{a}$ & $57.0 \mathrm{~b}$ & $204.0 \mathrm{a}$ & $67.2 \mathrm{a}$ & $108.7 \mathrm{a}$ \\
\hline 83 ppm 2.5-6.0 cm, + 2 WAFT; 125 ppm 4, 6 WAFT & $148.4 \mathrm{c}$ & $155 \mathrm{a}$ & $151.8 \mathrm{a}$ & $288.3 \mathrm{a}$ & $71.0 \mathrm{a}$ & $51.5 \mathrm{~b}$ & $193.0 \mathrm{a}$ & $89.0 \mathrm{a}$ & $103.2 \mathrm{a}$ \\
\hline $125 \mathrm{ppm} 2.5-6.0 \mathrm{~cm},+4,8$ WAFT & $162.4 \mathrm{bc}$ & $153 \mathrm{a}$ & $108.7 \mathrm{a}$ & $288.3 \mathrm{a}$ & $73.8 \mathrm{a}$ & $51.5 \mathrm{~b}$ & $198.5 \mathrm{a}$ & $79.7 \mathrm{a}$ & $215.2 \mathrm{a}$ \\
\hline $125 \mathrm{ppm} 4,8,12$ WAFT & $179.0 \mathrm{ab}$ & $165 \mathrm{a}$ & $174.0 \mathrm{a}$ & $286.8 \mathrm{a}$ & $60.6 \mathrm{a}$ & $74.2 \mathrm{ab}$ & $229.1 \mathrm{a}$ & $53.6 \mathrm{a}$ & $186.8 \mathrm{a}$ \\
\hline
\end{tabular}

${ }^{2}$ First treatment was applied at 2.5 to $6.0 \mathrm{~cm}$ of new shoot growth. Subsequent applications were made at the same or different dosage at the indicated number of weeks after first treatment (WAFT). Where shoot length is not indicated, treatments were made at the later timing indicated relative to 2.5 to 6.0 cm treatment.

${ }^{y}$ Values followed by the same letter are not significantly different $(P>0.05)$ according to the Waller-Duncan Bayesian k ratio test $(P>0.05)$.

${ }^{\mathrm{x}}$ Average number of flower clusters per tree in 2001 following treatment in 2000.

Table 3. Effect of various treatment dosages and timings of prohexadione-calcium applications on fruit weight, crop load, and return bloom in three pear cultivars, Hood River, Ore., 1999.

\begin{tabular}{|c|c|c|c|c|c|c|c|c|c|}
\hline \multirow[b]{2}{*}{ Treatment $^{\mathrm{z}}$} & \multicolumn{3}{|c|}{ Bartlett } & \multicolumn{3}{|c|}{ Bosc } & \multicolumn{3}{|c|}{ Anjou } \\
\hline & $\begin{array}{l}\text { Fruit wt } \\
(\mathrm{g})^{\mathrm{y}}\end{array}$ & $\begin{array}{c}\text { Fruit/ } \\
\text { tree }\end{array}$ & $\begin{array}{l}\text { Return } \\
\text { bloom }^{\mathrm{x}}\end{array}$ & $\begin{array}{l}\text { Fruit wt } \\
\text { (g) }\end{array}$ & $\begin{array}{c}\text { Fruit/ } \\
\text { tree }\end{array}$ & $\begin{array}{l}\text { Return } \\
\text { bloom }\end{array}$ & $\begin{array}{l}\text { Fruit wt } \\
(\mathrm{g})\end{array}$ & $\begin{array}{c}\text { Fruit/ } \\
\text { tree }\end{array}$ & $\begin{array}{l}\text { Return } \\
\text { bloom }\end{array}$ \\
\hline Untreated & $157.8 \mathrm{a}$ & $40.8 \mathrm{a}$ & $600.0 \mathrm{a}$ & $221.9 \mathrm{a}$ & $3.8 \mathrm{a}$ & $141.0 \mathrm{a}$ & $--^{w}$ & --- & $293.0 \mathrm{a}$ \\
\hline $250 \mathrm{ppm} 2.5-6.0 \mathrm{~cm}$ & $154.5 \mathrm{a}$ & $60.5 \mathrm{a}$ & $358.3 \mathrm{~d}$ & $211.9 \mathrm{a}$ & $1.8 \mathrm{a}$ & $78.0 \mathrm{bc}$ & --- & --- & $110.0 \mathrm{bc}$ \\
\hline $125 \mathrm{ppm} 2.5-6.0 \mathrm{~cm}+2 \mathrm{WAFT}$ & $162.4 \mathrm{a}$ & $67.3 \mathrm{a}$ & $566.8 \mathrm{ab}$ & $193.7 \mathrm{a}$ & $4.5 \mathrm{a}$ & $79.3 \mathrm{bc}$ & --- & --- & $55.3 \mathrm{c}$ \\
\hline $250 \mathrm{ppm} 2.5-6.0 \mathrm{~cm}+2 \mathrm{WAFT}$ & $179.5 \mathrm{a}$ & $28.8 \mathrm{a}$ & $482.5 \mathrm{c}$ & $233.5 \mathrm{a}$ & $4.5 \mathrm{a}$ & $49.3 \mathrm{~d}$ & --- & --- & $80.0 \mathrm{bc}$ \\
\hline $125 \mathrm{ppm} 2.5-6.0 \mathrm{~cm}+2,4,6$ WAFT & $179.4 \mathrm{a}$ & $53.3 \mathrm{a}$ & $509.0 \mathrm{bc}$ & $203.0 \mathrm{a}$ & $5.0 \mathrm{a}$ & $44.5 \mathrm{~d}$ & --- & --- & $72.5 \mathrm{bc}$ \\
\hline $125 \mathrm{ppm} 2.5-6.0 \mathrm{~cm}+4 \mathrm{WAFT}$ & $182.7 \mathrm{a}$ & $61.3 \mathrm{a}$ & $465.0 \mathrm{c}$ & $170.7 \mathrm{a}$ & $7.8 \mathrm{a}$ & $54.8 \mathrm{~cd}$ & --- & --- & $80.0 \mathrm{bc}$ \\
\hline $125 \mathrm{ppm} 2.5-6.0 \mathrm{~cm} ; 250 \mathrm{ppm} 4 \mathrm{WAFT}$ & $171.5 \mathrm{a}$ & $34.5 \mathrm{a}$ & $450.5 \mathrm{c}$ & $182.4 \mathrm{a}$ & $5.8 \mathrm{a}$ & $62.0 \mathrm{~cd}$ & --- & --- & $69.5 \mathrm{bc}$ \\
\hline $83 \mathrm{ppm} 2.5-6.0 \mathrm{~cm},+2,4,6,8$ WAFT & $184.2 \mathrm{a}$ & $68.0 \mathrm{a}$ & $587.8 \mathrm{a}$ & $166.5 \mathrm{a}$ & $2.5 \mathrm{a}$ & $91.0 \mathrm{~b}$ & --- & --- & $171.5 \mathrm{~b}$ \\
\hline
\end{tabular}

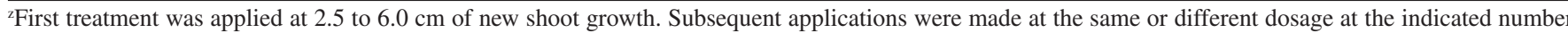
of weeks after first treatment (WAFT).

${ }^{y}$ Values followed by the same letter are not significantly different $(P>0.05)$ according to Fisher's protected LSD test $(P>0.05)$.

${ }^{x}$ Average number of flower clusters per tree in 2000 following treatment in 1999.

${ }^{w}$ Anjou trees were nonbearing in 1999.

Table 4. Effect of various treatment dosages and timings of prohexadione-calcium applications on fruit weight, crop load, and return bloom in three pear cultivars, Hood River, Ore., 2000.

\begin{tabular}{|c|c|c|c|c|c|c|c|c|c|}
\hline \multirow[b]{2}{*}{ Treatment $^{2}$} & \multicolumn{3}{|c|}{ Bartlett } & \multicolumn{3}{|c|}{ Bosc } & \multicolumn{3}{|c|}{ Anjou } \\
\hline & $\begin{array}{c}\text { Fruit wt } \\
(\mathrm{g})^{\mathrm{y}}\end{array}$ & $\begin{array}{l}\text { Fruit/ } \\
\text { tree }\end{array}$ & $\begin{array}{l}\text { Return } \\
\text { bloom }^{\mathrm{x}}\end{array}$ & $\begin{array}{c}\text { Fruit wt } \\
(\mathrm{g})\end{array}$ & $\begin{array}{c}\text { Fruit/ } \\
\text { tree }\end{array}$ & $\begin{array}{l}\text { Return } \\
\text { bloom }\end{array}$ & $\begin{array}{c}\text { Fruit wt } \\
(\mathrm{g})\end{array}$ & $\begin{array}{c}\text { Fruit/ } \\
\text { tree }\end{array}$ & $\begin{array}{l}\text { Return } \\
\text { bloom }\end{array}$ \\
\hline Untreated & $170.8 \mathrm{a}$ & $201.0 \mathrm{a}$ & $179.3 \mathrm{a}$ & $245.6 \mathrm{a}$ & $67.0 \mathrm{a}$ & $133.3 \mathrm{a}$ & $233.5 \mathrm{a}$ & $47.3 \mathrm{a}$ & $251.8 \mathrm{a}$ \\
\hline $83 \mathrm{ppm} 2.5-6.0 \mathrm{~cm},+2,4,6,8$ WAFT & $159.4 \mathrm{a}$ & $184.5 \mathrm{a}$ & $243.5 \mathrm{a}$ & $221.4 \mathrm{a}$ & $119.3 \mathrm{a}$ & $112.8 \mathrm{ab}$ & $230.9 \mathrm{a}$ & $54.8 \mathrm{a}$ & $181.8 \mathrm{a}$ \\
\hline 83 ppm $2.5-6.0 \mathrm{~cm},+2$ WAFT; 125 ppm 4, 6 WAFT & $178.1 \mathrm{a}$ & $231.0 \mathrm{a}$ & $148.0 \mathrm{a}$ & $228.6 \mathrm{a}$ & $85.8 \mathrm{a}$ & $64.8 \mathrm{~b}$ & 229.6 a & $78.3 \mathrm{a}$ & $188.0 \mathrm{a}$ \\
\hline 125 ppm $2.5-6.0 \mathrm{~cm},+4,8$ WAFT & $180.5 \mathrm{a}$ & $167.0 \mathrm{a}$ & $206.0 \mathrm{a}$ & $247.6 \mathrm{a}$ & $60.0 \mathrm{a}$ & $150.3 \mathrm{a}$ & $206.2 \mathrm{a}$ & $38.3 \mathrm{a}$ & $214.0 \mathrm{a}$ \\
\hline $125 \mathrm{ppm} \mathrm{4,} \mathrm{8,} 12$ WAFT & $163.0 \mathrm{a}$ & $179.8 \mathrm{a}$ & $207.5 \mathrm{a}$ & $246.3 \mathrm{a}$ & $94.3 \mathrm{a}$ & $123.0 \mathrm{a}$ & $220.1 \mathrm{a}$ & $98.0 \mathrm{a}$ & $293.5 \mathrm{a}$ \\
\hline
\end{tabular}

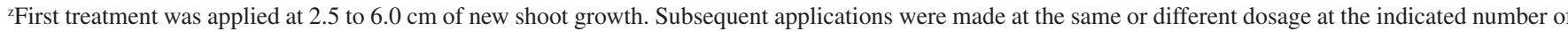
weeks after first treatment (WAFT). Where shoot length is not indicated, treatments were made at the later timing indicated relative to 2.5 to $6.0 \mathrm{~cm}$ treatment. yValues followed by the same letter are not significantly different $(P>0.05)$ according to Fisher's protected LSD test test $(P>0.05)$.

${ }^{x}$ Average number of flower clusters per tree in 2001 following treatment in 2000.

In Medford, return bloom was not affected by P-Ca treatments in 'Bartlett' or 'Red Anjou' pears, but return bloom decreased following all P-Ca treatments in 'Bosc' in both years of study except for 125 ppm applied only at 4,8 , and 12
WAFT (Tables 1 and 2). In Hood River, return bloom was reduced in all varieties following all treatments in 1999 except for $125 \mathrm{ppm}$ at 2.5 to $6.0 \mathrm{~cm}$ growth plus 2 WAFT and $83 \mathrm{ppm}$ at 2.5 to $6.0 \mathrm{~cm}$ growth plus $2,4,6$, and 8 WAFT in 'Bartlett' (Table 3). However, return bloom decreased following treatments applied in 2000 in Hood River only in 'Bosc', and only where $83 \mathrm{ppm}$ was applied at 2.5 to $6.0 \mathrm{~cm}$ growth plus 2 WAFT followed by $125 \mathrm{ppm}$ at 4 and 6 
Table 5. Effect of various treatment dosages and timings of prohexadione-calcium applications on fruit weight, crop load, and return bloom in three pear cultivars. Cashmere, Washington, 1999.

\begin{tabular}{|c|c|c|c|c|c|c|c|c|c|c|c|}
\hline \multirow[b]{2}{*}{ Treatment $^{2}$} & \multicolumn{3}{|c|}{ Bartlett } & \multirow[b]{2}{*}{ Treatment } & \multicolumn{3}{|c|}{ Bosc } & \multirow[b]{2}{*}{ Treatment } & \multicolumn{3}{|c|}{ Anjou } \\
\hline & $\begin{array}{l}\text { Fruit wt } \\
(\mathrm{g})^{\mathrm{y}}\end{array}$ & $\begin{array}{c}\text { Fruit/ } \\
\text { tree }\end{array}$ & $\begin{array}{l}\text { Return } \\
\text { bloom }^{\mathrm{x}}\end{array}$ & & $\begin{array}{c}\text { Fruit wt } \\
(\mathrm{g})\end{array}$ & $\begin{array}{c}\text { Fruit/ } \\
\text { tree }\end{array}$ & $\begin{array}{l}\text { Return } \\
\text { bloom }\end{array}$ & & $\begin{array}{c}\text { Fruit wt } \\
(\mathrm{g})\end{array}$ & $\begin{array}{c}\text { Fruit/ } \\
\text { tree }\end{array}$ & $\begin{array}{l}\text { Return } \\
\text { bloom }\end{array}$ \\
\hline Untreated & $217 \mathrm{a}$ & $75 \mathrm{a}$ & $12.7 \mathrm{a}$ & Untreated & $--^{w}$ & --- & $271 \mathrm{a}$ & Untreated & $238 \mathrm{a}$ & $235 \mathrm{a}$ & $8.8 \mathrm{a}$ \\
\hline $300 \mathrm{ppm} \mathrm{4,} 8$ WAFT & 194 a & $71 \mathrm{a}$ & $13.9 \mathrm{a}$ & $125 \mathrm{ppm} 2.5-6.0 \mathrm{~cm},+4 \mathrm{WAFT}$ & -- & -- & $176 \mathrm{bc}$ & $125 \mathrm{ppm} \mathrm{2,6,10 \textrm {WAFT }}$ & $222 \mathrm{a}$ & $290 \mathrm{a}$ & $5.9 \mathrm{a}$ \\
\hline \multirow[t]{3}{*}{600 ppm 8 WAFT } & $207 \mathrm{a}$ & $67 \mathrm{a}$ & $15.4 \mathrm{a}$ & $250 \mathrm{ppm} 2.5-6.0 \mathrm{~cm},+4 \mathrm{WAFT}$ & --- & --- & $128 \mathrm{c}$ & 125 ppm 6,10 WAFT & $221 \mathrm{a}$ & $291 \mathrm{a}$ & $6.4 \mathrm{a}$ \\
\hline & & & & 125 ppm 4, 8 WAFT & --- & --- & $231 \mathrm{ab}$ & & & & \\
\hline & & & & $250 \mathrm{ppm} 4,8 \mathrm{WAFT}$ & --- & --- & $177 \mathrm{bc}$ & & & & \\
\hline
\end{tabular}

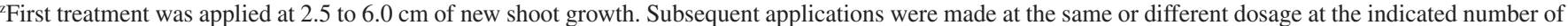
weeks after first treatment (WAFT). Where shoot length is not indicated, treatments were made at the later timing indicated relative to 2.5 to $6.0 \mathrm{~cm}$ treatment. ${ }^{y}$ Values followed by the same letter are not significantly different according to the Waller-Duncan Bayesian k ratio test $(P>0.05)$.

${ }^{x}$ Flower clusters per square centimeter limb cross-sectional area (Bartlett, Anjou) or per tree (Bosc) in 2000 following treatments in 1999.

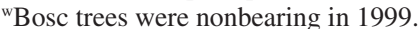

Table 6. Effect of various treatment dosages and timings of prohexadione-calcium applications on fruit weight, crop load, and return bloom in three pear cultivars, Cashmere, Wash., 2000.

\begin{tabular}{|c|c|c|c|c|c|c|c|c|c|}
\hline \multirow[b]{2}{*}{ Treatment ${ }^{2}$} & \multicolumn{3}{|c|}{ Bartlett } & \multicolumn{3}{|c|}{ Bosc } & \multicolumn{3}{|c|}{ Anjou } \\
\hline & $\begin{array}{l}\text { Fruit wt } \\
(\mathrm{g})^{\mathrm{y}}\end{array}$ & $\begin{array}{c}\text { Fruit/ } \\
\text { tree }\end{array}$ & $\begin{array}{l}\text { Return } \\
\text { bloom }^{\mathrm{x}}\end{array}$ & $\begin{array}{l}\text { Fruit wt } \\
(\mathrm{g})\end{array}$ & $\begin{array}{c}\text { Fruit/ } \\
\text { tree }\end{array}$ & $\begin{array}{l}\text { Return } \\
\text { bloom }\end{array}$ & $\begin{array}{l}\text { Fruit wt } \\
\text { (g) }\end{array}$ & $\begin{array}{c}\text { Fruit/ } \\
\text { tree }\end{array}$ & $\begin{array}{l}\text { Return } \\
\text { bloom }\end{array}$ \\
\hline Untreated & $180 \mathrm{a}$ & $196 \mathrm{a}$ & $5.0 \mathrm{a}$ & $230 \mathrm{a}$ & $180 \mathrm{a}$ & $2.0 \mathrm{a}$ & $210 \mathrm{a}$ & $173 \mathrm{a}$ & $8.1 \mathrm{a}$ \\
\hline $83 \mathrm{ppm} 2.5-6.0 \mathrm{~cm},+2,4,6 \mathrm{WAFT}$ & $150 \mathrm{~b}$ & $192 \mathrm{a}$ & $4.3 \mathrm{a}$ & $200 \mathrm{~b}$ & $200 \mathrm{a}$ & $0.6 \mathrm{~b}$ & $170 \mathrm{bc}$ & $192 \mathrm{a}$ & $5.2 \mathrm{a}$ \\
\hline $83 \mathrm{ppm}$ 2.5-6.0 cm, + 2 WAFT; $125 \mathrm{ppm} 4,6$ WAFT & $160 \mathrm{ab}$ & $223 \mathrm{a}$ & $5.0 \mathrm{a}$ & $200 \mathrm{~b}$ & $172 \mathrm{a}$ & $1.0 \mathrm{ab}$ & $190 \mathrm{ab}$ & $175 \mathrm{a}$ & $6.0 \mathrm{a}$ \\
\hline $125 \mathrm{ppm} 2.5-6.0 \mathrm{~cm},+4$ WAFT & $170 \mathrm{ab}$ & $216 \mathrm{a}$ & $4.6 \mathrm{a}$ & $200 \mathrm{~b}$ & $194 \mathrm{a}$ & $1.4 \mathrm{ab}$ & $190 \mathrm{ab}$ & $189 \mathrm{a}$ & $6.2 \mathrm{a}$ \\
\hline 125 ppm 4 WAFT & $170 \mathrm{ab}$ & $216 \mathrm{a}$ & $4.4 \mathrm{a}$ & $210 \mathrm{ab}$ & $151 \mathrm{a}$ & $1.0 \mathrm{ab}$ & $200 \mathrm{a}$ & $178 \mathrm{a}$ & $7.2 \mathrm{a}$ \\
\hline $125 \mathrm{ppm} 2.5-6.0 \mathrm{~cm},+2,4,6$ WAFT & $150 \mathrm{~b}$ & $218 \mathrm{a}$ & $4.8 \mathrm{a}$ & $200 \mathrm{~b}$ & $175 \mathrm{a}$ & $0.4 \mathrm{~b}$ & $150 \mathrm{c}$ & $170 \mathrm{a}$ & $6.2 \mathrm{a}$ \\
\hline
\end{tabular}

${ }^{2}$ First treatment was applied at 2.5 to $6.0 \mathrm{~cm}$ of new shoot growth. Subsequent applications were made at the same or different dosage at the indicated number of weeks after first treatment (WAFT). Where shoot length is not indicated, treatments were made at the later timing indicated relative to 2.5 to $6.0 \mathrm{~cm}$ treatment. ${ }^{y}$ Values followed by the same letter are not significantly different according to the Waller-Duncan Bayesian k ratio test $(P>0.05)$.

xFlower clusters per square centimeter limb cross-sectional area in 2001 following treatments in 2000.

WAFT (Table 4). In Cashmere, return bloom was affected only in 'Bosc'. Return bloom was reduced following treatments to nonbearing trees in 1999 of 125 or $250 \mathrm{ppm}$ at 2.5 to 6.0 $\mathrm{cm}$ growth plus 4 WAFT, and $250 \mathrm{ppm}$ at 2.5 to $6.0 \mathrm{~cm}$ growth plus 4 and 8 WAFT (Table 5). In 2001, 'Bosc' return bloom was reduced following treatments of 83 or $125 \mathrm{ppm}$ at 2.5 to $6.0 \mathrm{~cm}$ growth plus 2, 4, and 6 WAFT (Table 6). In the 2000 trial in Cashmere, rootstock had no interactive effect on P-Ca treatments in terms of fruit development or return bloom (data not shown).

\section{Discussion}

P-Ca inhibits biosynthesis of growthactive gibberellins (GAs) at a late stage in GA biosynthesis (Evans et al., 1999). P-Ca degrades relatively quickly, with a half-life in higher plants of a few weeks (Evans et al., 1999). P-Ca has been effective in reducing pear shoot growth following applications during early shoot growth, but shoot growth may resume later in the season (Elfving et al., 2002, 2003b).

Applied GAs (e.g., $\mathrm{GA}_{3}$ or $\mathrm{GA}_{4+7}$ ) may inhibit flower bud development in several fruit tree species (Hoad, 1984). GA applied to 'Bartlett' pears at bloom or petal fall reduced development of flower buds for the following year (Griggs and Iwakiri, 1961). GAs diffusing from developing pome fruit seeds are capable of reducing flower bud development (Chan and Cain, 1967). Greater amounts of GAs were found diffusing from seeds of apple cultivars prone to biennial bearing than in those cultivars which do not exhibit biennial bearing (Ebert and Bangerth, 1981). More diffusible GA-like substance diffused from seeded 'Winter Nelis' than from seeded or unseeded 'Bartlett' pears in California during the postbloom period (Gil et al., 1973). The presence of seeded fruits inhibited flower bud formation in 'Winter Nelis' to a greater extent than in 'Bartlett' (Griggs et al., 1970).

Since GAs may function in inhibition of flower bud development, inhibitors of GA synthesis may be expected to increase flower bud formation. Applications of daminozide, a GA inhibitor which, like $\mathrm{P}-\mathrm{Ca}$, mimics 2-oxoglutaric acid (Rademacher, 2000), increased return bloom in 'Northern Spy' apple (Elfving and Cline, 1990). P-Ca treatments increased flower bud formation in 'Macoun' apple, but not in 'Delicious' or 'Fuji' (Owen and Stover, 1999).

The reduction of return bloom observed in 'Bosc' pear associated with P-Ca treatments in this study appears to be paradoxical. However, the effect may be related to a combination of factors that distinguish pear from apple and 'Bosc' from the other cultivars in this study, as well as the relatively short life of P-Ca in planta.

Inhibition of growth-active GAs in plant tissues by $\mathrm{P}-\mathrm{Ca}$ results in the accumulation of precursor molecules (Evans et al., 1999; Rademacher, 2000). Accumulation of precursors may allow for relatively rapid formation of growth-active GAs when biosynthesis is no longer inhibited by $\mathrm{P}-\mathrm{Ca}$. In turn, this may result in the strong late-summer growth sometimes observed in pear trees following early season growth suppression by P-Ca (Elfving et al., 2002, 2003b). In addition, P-Ca may protect endogenous active GAs from being metabolically inactivated (Rademacher, 2000).
'Bartlett' and 'Anjou' pears typically bear annual crops (Williams et al., 1978), while 'Bosc' tends toward biennial bearing, especially following a heavy seeded crop (Lombard, 1986). The tendency for biennial bearing may indicate greater sensitivity to inhibition of flower bud development by GAs. Furthermore, flower bud formation occurs relatively late in pear (up to 60 days after full bloom) as compared to apple (Westwood, 1993). It is conceivable that accumulated GA precursors or their derivatives, or late-formed growth-active GAs play a role in suppression of flower bud formation in the biennial-bearing prone 'Bosc', but do not function in this way in 'Bartlett', 'Anjou', or 'Red Anjou'.

Decreased fruit size in fruit trees is often a consequence of increased crop load and accompanying changes in leaf-to-fruit ratio (Westwood, 1993). In 'McIntosh' apples, decreased fruit weight following P-Ca treatments corresponded to increased fruit set (Greene, 1999). The gibberellic-acid biosynthesis inhibitor daminozide was shown to reduce apple fruit size by inhibition of cell division when applied early in the season (Martin et al., 1968). The reduction in fruit weight observed in 'Bartlett' pears at Medford in both years and in all cultivars at Cashmere in 2000 in the present study was apparently not associated with crop load, which did not differ significantly among treatments at any of the experimental sites. The direct effects which P-Ca may have on cell division or enlargement in pear fruitlets have yet to be measured.

\section{Literature Cited}

Basak, A. and W. Rademacher. 2000. Growth regulation of pome and stone fruit trees by use of 
prohexadione-Ca. Acta Hort. 514:41-50.

BASF Corp. 2003. Apogee plant growth regulator. 29 May 2003. http://www.agproducts.basf-corp. com/Products/Products.asp.

Byers, R.E. and K.S. Yoder. 1999. Prohexadionecalcium inhibits apple, but not peach, tree growth but has little influence on apple fruit thinning or quality. HortScience 34:1205-1209.

Chan, R.G. and J.C. Cain. 1967. The effect of seed formation on subsequent flowering in apple. J. Amer. Soc. Hort. Sci. 91:63-68.

Costa, G., A.M. Bregoli, E. Sabatini, B. Cristina, and V. Giannina. 2000. Apogee controls shoot growth and fruit parameters. HortScience 35:421 (abstr.).

Ebert, A. and F. Bangerth. 1981. Relations between the concentration of diffusable and extractable gibberellin-like substances and the alternate bearing behaviour in apple as affected by chemical fruit thinning. Scientia Hort. 15:45-52.

Elfving, D.C. and R.A. Cline. 1990. Growth and productivity of vigorous 'Northern Spy'/MM.106 apple trees in response to annually applied growth control techniques. J. Amer. Soc. Hort. Sci. 115:212-218.

Elfving, D.C., D. Sugar, and D. Faubion. 2002. Pear tree shoot growth patterns in relation to chemical control of vegetative growth with prohexadione-calcium (Apogee ${ }^{\mathrm{TM}}$ ). Acta Hort. 596:711-716.

Elfving, D.C., G.A. Lang, and D.B. Visser. 2003a. Prohexadione-Ca and ethephon reduce shoot growth and increase flowering in young, vigorous sweet cherry trees. HortScience 38:293-298.

Elfving, D.C., L. Lombardini, J.R. McFerson, S.R. Drake, D.F. Faubion, T.D. Auvil, G. Van Ee, and D.B. Visser. 2003b. Effects of directed applications of prohexadione-calcium to tops of mature pear trees on shoot growth, light penetration, pruning and fruit quality. J. Amer. Pom. Soc. 57:45-57.

Evans, J.R., R.R. Evans, C.L Regusci, and W. Rademacher. 1999. Mode of action, metabolism, and uptake of BAS $125 \mathrm{~W}$, prohexadione-Ca. HortScience 34:1200-1201.

Forshey, C.G. and D.C. Elfving. 1979. Branch samples for yield and fruit size comparisons in apple. HortScience 14:143-144.

Gil, G.F., G.C. Martin, and W.H. Griggs. 1973. Fruit-set and development in the pear: Diffusible growth substances from seeded and seedless fruits. J. Amer. Soc. Hort. Sci. 98:51-54.

Greene, D.W. 1999. Tree growth management and fruit quality of apple trees treated with prohexadione-Ca (BAS 125). HortScience 34:1209-1212.

Griggs, W.H. and B.T. Iwakiri. 1961. Effects of gibberellin and 2,4,trichlorophenoxypropionic acid sprays on 'Bartlett' pear trees. Proc. Amer. Sco. Hort. Sci. 77:73-89.

Griggs, W.H., G.C. Martin, and B.T. Iwakiri. 1970. The effect of seedless versus seeded fruit development on flower bud formation in pear. J. Amer. Soc. Hort. Sci. 95:243-248.
Hoad, G.V. 1984. Hormonal regulation of fruit-bud formation in fruit trees. Act Hort. 149:13-23.

Lombard P.B. 1987. Principal green and new pear varieties for USA, p. 45-67 in: Pear production in the Pacific Northwest: Proceedings of the 1986 Pacific Northwest Tree Fruit Shortcourse. Wash. State Univ., Pullman.

Martin, D., T.L. Lewis, and J. Cerny. 1968. The effect of alar on fruit cell division and other characteristics in apples. Proc. Amer. Soc. Hort. Sci. 92:67-70.

Owen, C.L. and E. Stover. 1999. Vegetative growth and flowering of young apple trees in response to prohexadione-calcium. HortScience 34:1194-1196.

Rademacher, W. 2000. Growth retardants: Effects on gibberellin biosynthesis and other metabolic pathways. Annu. Rev. Plant Physiol. Plant Mol. Biol. 512:501-531.

Theron, K.I., M. LeGrange, M. Smit, S. Reynolds, and G. Jacobs. 2002. Controlling vigor and color development in the bi-colored pear cultivar Rosemarie. Acta Hort. 596:753-756.

Unrath, C.R. 1999. Prohexadione-Ca: A promising chemical for controlling vegetative growth in apples. HortScience 34:1197-1200.

Westwood, M.N. 1993. Temperate zone pomology, $3^{\text {rd }}$ ed. Timber Press, Portland, Ore.

Williams, M.W., H.M. Couey, H. Moffitt, and D.L. Coyier. 1978. Pear production. USDA Hndbk. 526 